\title{
The use of US health insurance data for surveillance of rare disorders: hereditary hemorrhagic telangiectasia
}

\author{
Scott D. Grosse, PhD' ${ }^{1}$, Sheree L. Boulet, DrPH, MPH${ }^{2}$, Althea M. Grant, $\mathrm{PhD}^{1}$, Mary M. Hulihan, MPH \\ and Marie E. Faughnan, $\mathrm{MD}^{3-5}$
}

Purpose: To assess the utility of US health insurance data for surveillance of hereditary hemorrhagic telangiectasia, an autosomaldominant blood vasculature disorder with an estimated prevalence of 1.5-2.0 per 10,000 persons worldwide.

Methods: We used 2005-2010 MarketScan Research Databases to identify individuals with employer-sponsored health insurance and International Classification of Disease, 9th Revision, Clinical Modification codes of 448.0 present in either one inpatient claim or two outpatient claims 30 days apart to define hereditary hemorrhagic telangiectasia. We examined frequencies of International Classification of Disease, 9th Revision, Clinical Modification codes for conditions that are complications of hereditary hemorrhagic telangiectasia among individuals with hereditary hemorrhagic telangiectasia and the general population to identify combinations of codes associated with hereditary hemorrhagic telangiectasia.

Results: Excluding observations from one state, the average prevalence of hereditary hemorrhagic telangiectasia was 0.3 per 10,000 persons. The reported prevalence rose with age from $\sim 0.1$ per 10,000 at ages $<30$ years to $1.0-1.1$ per 10,000 at ages 70 years and above. The condition codes that were most specific to presumed hereditary hemorrhagic telangiectasia were lung arteriovenous malformations and upper gastrointestinal angiodysplasia. Combinations of those codes and codes for brain arteriovenous malformation and epistaxis were highly predictive of reporting of hereditary hemorrhagic telangiectasia, with $20-57 \%$ of enrollees with those codes also meeting the study definition for hereditary hemorrhagic telangiectasia.

Conclusion: Hereditary hemorrhagic telangiectasia is underrecognized in US administrative data. Administrative health data can be used to identify individuals with combinations of signs that are suggestive of hereditary hemorrhagic telangiectasia. Studies are needed to test the hypothesis that referral for evaluation of individuals with administrative records suggestive of undiagnosed hereditary hemorrhagic telangiectasia could lead to diagnosis and access to life-saving treatments for both them and affected family members.

Genet Med advance online publication 23 May 2013

Key Words: administrative data; epidemiology; Osler-WeberRendu disease; prevalence

\section{INTRODUCTION}

Rare disorders, defined in Europe as diseases affecting $<1$ per 2,000 people, are collectively an important public health issue but are typically underrecognized. A public health approach to rare disorders begins with assessments of the prevalence or incidence of these disorders and their impacts on health status, disability, mortality, and health services use. ${ }^{1}$

Surveillance for rare disorders is challenging. Populationbased surveys typically lack sufficient numbers of observations, and many rare diseases are underreported. The primary source of information is usually patient registries, whether based on clinical referral centers or organized by disease advocacy organizations based on patient self-referral, but findings may not be generalizable to the entire population. Active population-based surveillance can be conducted through canvassing and review of medical charts, ${ }^{2}$ but that approach is time consuming and costly.

Passive surveillance conducted through the review of administrative health-care data can cover large populations at very low cost. Administrative health databases generally identify conditions through the use of International Classification of Disease diagnostic codes, either the International Classification of Disease, 9th Revision (ICD-9), used in the United States, or the 10th Revision (ICD-10), used in the rest of the world (and for mortality statistics in the United States). Two major types of administrative data are discharge records from hospitals and claims records from health insurers, whether private or public payers. ${ }^{3}$ The disadvantage is that those records lack validated diagnostic and clinical information.

The focus of this study is one specific rare disorder, hereditary hemorrhagic telangiectasia (HHT), which is also known as Osler-Weber-Rendu or Rendu-Osler-Weber syndrome (OMIM no. 187300). HHT is an autosomal-dominant disorder of blood vasculature, with an estimated prevalence of up to 2.0 per 10,000 persons worldwide. ${ }^{4,5}$ Mutations in two genes, endoglin $(E N G)$ and activin A receptor type II-like 1 (ACVRL1), are associated with HHT1 and HHT2, respectively. Three less common forms of HHT also exist, including one form associated with juvenile polyposis syndrome caused by mutations in the

${ }^{1}$ Division of Blood Disorders, National Center on Birth Defects and Developmental Disabilities, Centers for Disease Control and Prevention, Atlanta, Georgia, USA; ${ }^{2}$ Division of Reproductive Health, National Center for Chronic Disease Prevention and Health Promotion, Centers for Disease Control and Prevention, Atlanta, Georgia, USA; ${ }^{3}$ Toronto HHT Centre, Division of Respirology, Department of Medicine, St Michael's Hospital, University of Toronto, Toronto, Ontario, Canada; ${ }^{4}$ Li Ka Shing Knowledge Institute, St Michael's Hospital, University of Toronto, Toronto, Ontario, Canada; ${ }^{5}$ HHT Foundation International, Monkton, Maryland, USA. Correspondence: Scott D. Grosse (sgrosse@cdc.gov) 
SMAD-related protein 4 gene (SMAD4) and HHT3 and HHT4, for which loci have been determined but the genes have not yet been identified. ${ }^{6}$

HHT is a highly penetrant disorder, with the most common manifestation of recurrent epistaxis developing in 95\% of individuals with HHT by the age of 50 years. ${ }^{7}$ HHT is characterized by the development of vascular malformations with direct artery-to-vein connection and loss of intervening capillaries. The smaller lesions, telangiectases, typically occur in the nasal, oral, or gastric mucosa or dermis of the hands or face, or in the liver. Chronic nasal or gastrointestinal (GI) bleeding is common and often results in the development of iron-deficiency anemia. The larger lesions, arteriovenous malformations (AVMs), typically occur in the lungs, brain, or liver and can cause life-threatening complications such as stroke, brain abscess, heart failure, and life-threatening hemorrhage. ${ }^{8}$ Although different forms of HHT are associated with varying frequencies and timing of manifestation of signs or symptoms, the manifestations listed above occur in all types of HHT.,10

The signs of HHT may go unrecognized and untreated for decades or cause premature death. ${ }^{11} \mathrm{~A}$ recent study from Italy involving 112 HHT patients who were index cases in their families reported that the mean age at which symptomatic disease manifested was 16.2 years, the mean age of referral for evaluation of those symptoms was 31.7 years, and the mean age of diagnosis of HHT was 45.9 years, for a mean delay of almost 30 years between onset of symptoms and diagnosis. ${ }^{12}$ HHT is associated with significantly higher rates of premature mortality before 60 years of age. ${ }^{4,13}$

A definite diagnosis of HHT is established according to the international consensus Curacao Criteria by the presence of three of four criteria: epistaxis, multiple telangiectases, visceral lesions, and a family history with one or more first-degree relatives affected. ${ }^{14,15}$ Once an HHT diagnosis is established, screening and preventative management of AVMs is recommended ${ }^{14}$ and available in HHT Centers of Excellence (http://www.hht. org). As such, establishment of a diagnosis of HHT has important clinical implications, both for prevention and appropriate clinical care.

The purposes of surveillance for rare disorders such as HHT include a better understanding of the frequency of the disorder and its complications by age, race/ethnicity, and geographic location. Surveillance information can be used to guide the provision of health-care services and to evaluate the effectiveness and quality of medical care and interventions. In addition, understanding the characteristics of people who have undiagnosed HHT may help to guide the development of protocols for identifying such individuals using administrative data and referring them for evaluation for potential HHT. Improved identification can lead to screening for AVMs and the use of potentially life-saving treatments. ${ }^{14}$

The current study has three purposes. First, it assesses the prevalence of reported HHT among the US population with employer-sponsored insurance. Second, it assesses the frequency of reported symptoms characteristic of HHT both among people meeting the study definition for HHT and those with no HHT diagnosis code. Third, it seeks to identify the most specific combinations of signs and symptoms that are predictive of presumed HHT. The ultimate goal is to promote earlier diagnosis of HHT by using algorithms of combinations of diagnosis codes to identify patients who could be referred for HHT evaluation.

\section{MATERIALS AND METHODS}

The data for this study were derived from the 2005-2010 MarketScan Commercial Claims and Encounters and MarketScan Medicare Supplemental Research Databases (Truven Health Analytics, formerly the health-care business of Thomson Reuters). These databases arise from the processing of health insurance claims for reimbursement and include information on the diagnoses as submitted by providers. The MarketScan Commercial Database contains records on employer-sponsored insurance for employees and their dependents. It includes data from large and mid-sized, self-insured employers and employers who purchase fully insured health plans on behalf of their employees. The 2010 MarketScan Commercial Database had records on 45 million individuals younger than 65 years. The MarketScan Supplemental Medicare Database contains records on retired employees and spouses older than 65 years who are enrolled in Medicare with supplemental Medigap insurance paid by their former employers. All health-care services for which claims were submitted are covered. The 2010 Supplemental Medicare database includes 3.7 million individuals older than 65 years.

The MarketScan Research Databases are composed of separate databases for each year for enrollment, inpatient services, outpatient (including hospital emergency or ambulatory services, clinical visits, laboratory tests, and home healthcare) services, and outpatient prescription medications for those enrollees with prescription drug coverage. An encrypted individual identifier can be used to link records across databases and years to identify all records for unique individuals. The inpatient claim records allowed for up to 15 different ICD-9, Clinical Modification (ICD-9-CM) diagnosis codes, and the outpatient claim records allowed for two diagnosis codes to be listed between 2005 and 2008 and four diagnosis codes during 2009-2010. In addition to ICD-9-CM codes, the inpatient and outpatient databases listed procedure codes. The drugs database listed National Drug Classification codes but not ICD-9-CM codes. The only demographic variables for enrollees were age and sex. Provider type and location (three-digit zip codes) were included for both inpatient and outpatient databases.

To identify cases of HHT, all records from 2005 through 2010 for individuals with prescription drug coverage were analyzed. Cases of potential HHT were identified through use of the 448.0 ICD-9-CM diagnosis code, which in principle is specific to HHT. Any individual with a code of 448.0 in an inpatient record was classified as a case of HHT. In addition, individuals with two or more outpatient claims containing the 448.0 code for service dates more than 30 days apart were classified as cases 
of HHT. It is a standard practice in health services research using claims data to require more than one outpatient claim with a given diagnosis code separated in time in order to minimize "rule-out diagnoses" associated with outpatient services such as laboratory tests or radiology examinations when no diagnosis is found, in addition to minimizing coding errors. ${ }^{16,17}$ Moreover, using multiple years of data for the same individuals improves the sensitivity of ascertaining cases of chronic diseases, which may go unrecorded in services during a given year. ${ }^{17}$

Geographic variations in the reported frequency of HHT diagnoses were assessed according to census geographic region (Northeast, North Central, South, and West), state, and metropolitan area of residence, with three-digit zip codes aggregated to define state and metropolitan area of residence. Prevalence rates across regions and states were compared to determine potential clusters of HHT cases that might reflect different geographic patterns of coding or potentially the presence of large kindreds.

The frequency of other diagnoses associated with manifestations or signs of HHT on an a priori basis was compared between the presumed HHT cases and the remainder of the sample, excluding individuals with just one outpatient claim with an ICD-9-CM code of $448.0(n=4,438)$, to identify those diagnoses that were most closely associated with presumed HHT. The other diagnoses comprised relatively specific signs of HHT as follows: epistaxis (784.7), telangiectasia (448.9), brain AVM (747.81), lung AVM (747.3, 417.0), unspecified AVM of the peripheral vasculature system (747.6), angiodysplasia of the upper GI tract (537.82, 537.83), and angiodysplasia of the intestine $(569.84,569.85)$. The following codes were considered by expert opinion to be potential but less specific markers of HHT: anemia: $(280,285)$, aneurysm or intracerebral hemorrhage (437.3; 431), acquired arteriovenous fistula (447.0), hemoptysis (786.3), and hemorrhage of the GI tract, unspecified (578.9). Although aneurysm is not a complication of HHT, the code may be miscoded to refer to a brain AVM.

Because recurrent epistaxis and the presence of multiple AVMs are expected to be more common among people with HHT, it was hypothesized that the presence of multiple claims for those signs would be higher among people meeting the study definition for presumed HHT. However, although multiple claims separated by time are likely to reflect multiple episodes, it is not possible using claims data to distinguish multiple from single AVMs diagnosed on one occasion.

\section{RESULTS}

During 2010, data on a total of $37,463,423$ enrollees in the Commercial and Supplemental Medicare databases with prescription coverage were analyzed, of which 1,496 met the case definition for presumed HHT based on ICD-9-CM codes using records from 2005 through 2010. An additional 4,438 individuals had just one outpatient claim with a code of 448.0 during that period and were excluded from further analysis.

The overall prevalence of presumed HHT in the preliminary analysis was 0.40 per 10,000 persons, which varied by region from 0.32 in the South to 0.40 in the West with a much higher prevalence of 0.56 in the North Central region (data not shown). The higher prevalence in the North Central region was concentrated in one state, Michigan, which had the highest apparent prevalence of presumed HHT, 2.00 per 10,000, accounting for $19.6 \%$ of presumed HHT cases and 3.9\% of the MarketScan sample. Further analysis revealed that few HHT cases in Michigan had diagnostic codes for manifestations or signs of HHT. To avoid potential bias, all records from Michigan were excluded from further analyses.

In the final data set, there were 1,203 HHT cases among 35,459,534 enrollees. The prevalence of presumed HHT in the North Central region (excluding Michigan) of 0.31 per 10,000 was similar to the overall prevalence of 0.33 per 10,000 (Table 1).

As expected, the estimated prevalence of HHT increased with age (Table 1). It was $\sim 0.1$ per 10,000 below 30 years, reached 0.53 at $50-59$ years, and in the two oldest age groups, $70-79$ and 80 years and older, it was 1.14 and 1.04 per 10,000, respectively.

The frequencies and corresponding rate ratios of potential signs of HHT recorded among individuals with and without HHT are displayed in Table 2. The most common coexisting condition reported was anemia, which was reported for $50.0 \%$ of people with presumed HHT. Anemia is fairly common in the general population, reported for $5.6 \%$ of those without presumed HHT, and the resulting ratio is a relatively modest 9:1.

Table 1 Prevalence of presumed HHT (ICD-9-CM code 448.0) in MarketScan Commercial and Supplemental Medicare Databases, 2005-2010, by age, sex, and region (excluding Michigan)

\begin{tabular}{lccc} 
& HHT $(\boldsymbol{n})$ & $\begin{array}{c}\text { Population } \\
(\boldsymbol{n})\end{array}$ & $\begin{array}{c}\text { Prevalence } \\
\text { per 10,000 }\end{array}$ \\
\hline Age (years) & 55 & $4,663,310$ & 0.12 \\
$0-9$ & 47 & $5,062,392$ & 0.09 \\
$10-19$ & 58 & $4,646,957$ & 0.12 \\
$20-29$ & 113 & $5,306,579$ & 0.21 \\
30-39 & 203 & $5,914,142$ & 0.34 \\
$40-49$ & 305 & $5,737,111$ & 0.53 \\
50-59 & 237 & $2,983,016$ & 0.79 \\
60-69 & 116 & $1,014,399$ & 1.14 \\
$70-79$ & 69 & 665,215 & 1.04 \\
$\geq 80$ & & & \\
Sex & 474 & $17,347,778$ & 0.27 \\
Male & 729 & $18,645,343$ & 0.39 \\
\hline Female & & & \\
\hline Region & 173 & $5,256,430$ & 0.33 \\
Northeast & 266 & $8,491,129$ & 0.31 \\
North Central & 468 & $14,648,998$ & 0.32 \\
South & 294 & $7,429,173$ & 0.40 \\
\hline West & & & \\
\hline
\end{tabular}

$\mathrm{HHT}$, hereditary hemorrhagic telangiectasia; ICD-9-CM, International Classification of Disease, 9th Revision, Clinical Modification. 
Table 2 Frequency of presence of ICD-9-CM codes for signs of HHT recorded among individuals with and without ICD-9-CM code 448.0 in MarketScan Commercial and Supplemental Medicare Databases, 2005-2010 (excluding Michigan)

\begin{tabular}{|c|c|c|c|c|c|}
\hline Condition & $\begin{array}{l}\text { With } \\
\text { HHT (N) }\end{array}$ & $\%$ & $\begin{array}{l}\text { Without } \\
\text { HHT (n) }\end{array}$ & $\%$ & $\begin{array}{l}\text { Rate } \\
\text { ratio }\end{array}$ \\
\hline Epistaxis & 411 & 34.2 & 294,938 & 0.82 & 42 \\
\hline Brain AVM & 65 & 5.4 & 15,079 & 0.04 & 135 \\
\hline Lung AVM & 173 & 14.4 & 10,285 & 0.03 & 480 \\
\hline $\begin{array}{l}\text { Unspecified AVM of } \\
\text { vascular system }\end{array}$ & 152 & 12.6 & 17,441 & 0.05 & 252 \\
\hline $\begin{array}{l}\text { Arteriovenous } \\
\text { fistula, acquired }\end{array}$ & 9 & 0.8 & 5,005 & 0.01 & 80 \\
\hline Any AVM & 285 & 23.7 & 45,600 & 0.13 & 182 \\
\hline $\begin{array}{l}\text { Upper Gl } \\
\text { angiodysplasia }\end{array}$ & 97 & 8.1 & 7,613 & 0.02 & 405 \\
\hline $\begin{array}{l}\text { Angiodysplasia of } \\
\text { the intestine }\end{array}$ & 85 & 7.1 & 15,606 & 0.04 & 178 \\
\hline $\begin{array}{l}\text { Hemorrhage of Gl } \\
\text { tract, unspecified }\end{array}$ & 212 & 17.6 & 274,404 & 0.76 & 23 \\
\hline Anemia & 602 & 50.0 & $1,997,615$ & 5.55 & 9 \\
\hline $\begin{array}{l}\text { Aneurysm or } \\
\text { intracerebral } \\
\text { hemorrhage }\end{array}$ & 35 & 2.9 & 54,313 & 0.15 & 19 \\
\hline Hemoptysis & 46 & 3.8 & 72,879 & 0.20 & 19 \\
\hline Dyspnea & 382 & 31.8 & $3,143,068$ & 8.73 & 4 \\
\hline Telangiectasia & 120 & 10.0 & 43,849 & 0.12 & 83 \\
\hline
\end{tabular}

AVM, arteriovenous malformation; Gl, gastrointestinal; $\mathrm{HHT}$, hereditary hemorrhagic telangiectasia; ICD-9-CM, International Classification of Disease, 9th Revision, Clinical Modification.
Epistaxis was reported during 2008 for $34.2 \%$ of people with HHT but for only $0.8 \%$ of others, resulting in a ratio of $42: 1$. The most specific signs of HHT were lung AVM, with a ratio of 480:1, and upper GI angiodysplasia, with a ratio of 4,051:1, but these were reported for only 14.4 and $8.1 \%$ of those with HHT, respectively. Other relatively specific diagnoses with ratios $>100: 1$ were unspecified AVM of the peripheral vasculature system (252:1), angiodysplasia of the intestine (178:1), and brain AVM (135:1).

The proportions of individuals with multiple claims for epistaxis, lung AVM, or brain AVM among those with any claim were only slightly higher among those with HHT $(83.5,78.8$, and $70.5 \%$, respectively) than those without an HHT diagnosis (61.0, 55.7, and $64.0 \%$, respectively) (data not shown).

The frequencies of signs reported among those with HHT by broad age categories are depicted in Table 3. The most marked finding is that GI bleeding and anemia are uncommon before the age of 30 years among people with HHT but are increasingly common with advancing age. Conversely, a larger proportion of younger adults with HHT are recorded as having AVMs. Among adults older than 60 years with HHT, 18.7\% had any type of AVM recorded on claims during 2010, as compared with $32.5 \%$ of those younger than 30 years.

Information on the frequency of pairwise combinations of signs is reported in Table 4, except that for angiodysplasia of the intestine only the interaction with lung AVM is reported. The six most specific combinations involve either lung AVM together with either upper GI angiodysplasia (rate ratio of 39,992), brain AVM (rate ratio of 17,997), angiodysplasia of the intestine (rate ratio of 15,426), other AVM (rate ratio of 12,665 ), or epistaxis (rate ratio of 7,791) or the combination of upper GI angiodysplasia with brain AVM (rate ratio of 7,831).

Table 3 Frequency by age of presence of ICD-9-CM codes for signs of HHT recorded among individuals with HHT (ICD-9-CM 448.0) in MarketScan Commercial and Supplemental Medicare Databases, 2005-2010 (excluding Michigan)

\begin{tabular}{|c|c|c|c|c|c|c|}
\hline \multirow[b]{2}{*}{ Condition } & \multicolumn{2}{|c|}{$<30$ years $(n=160)$} & \multicolumn{2}{|c|}{$30-59$ years $(n=621)$} & \multicolumn{2}{|c|}{$\geq 60$ years $(n=422)$} \\
\hline & $n$ & $\%$ & $n$ & $\%$ & $n$ & $\%$ \\
\hline Epistaxis & 30 & 18.8 & 209 & 33.7 & 172 & 40.8 \\
\hline Lung AVM & 33 & 20.6 & 99 & 15.9 & 41 & 9.7 \\
\hline Unspecified AVM of vascular system & 18 & 11.3 & 82 & 13.2 & 52 & 12.3 \\
\hline Upper GI angiodysplasia & 1 & 0.6 & 49 & 7.9 & 47 & 11.1 \\
\hline Angiodysplasia of the intestine & 2 & 1.3 & 39 & 6.3 & 44 & 10.4 \\
\hline Hemorrhage of GI tract, unspecified & 8 & 5.0 & 86 & 13.9 & 118 & 28.0 \\
\hline Anemia & 23 & 14.4 & 304 & 49.0 & 275 & 65.2 \\
\hline Telangiectasia & 17 & 10.6 & 57 & 9.2 & 46 & 10.9 \\
\hline
\end{tabular}

AVM, arteriovenous malformation; GI, gastrointestinal; HHT, hereditary hemorrhagic telangiectasia; ICD-9-CM, International Classification of Disease, 9th Revision, Clinical Modification. 
Table 4 Frequency of presence of combinations of ICD-9-CM codes suggestive of HHT recorded among individuals with and without ICD-9-CM code 448.0 in MarketScan Commercial and Supplemental Medicare Databases, 2005-2010 (excluding Michigan)

\begin{tabular}{|c|c|c|c|c|c|}
\hline Condition & With HHT code $(n)$ & $\%$ & Without HHT code $(n)$ & $\%$ & Rate ratio \\
\hline Epistaxis + brain AVM & 27 & 2.2 & 347 & 0.001 & 2,282 \\
\hline Epistaxis + lung AVM & 60 & 5.0 & 231 & 0.001 & 7,791 \\
\hline Epistaxis + upper Gl angiodysplasia & 33 & 2.7 & 427 & 0.001 & 2,276 \\
\hline Epistaxis + telangiectasia & 59 & 4.9 & 847 & 0.002 & 2,082 \\
\hline Lung AVM + unspecified AVM of vascular system & 68 & 5.7 & 162 & 0.000 & 12,664 \\
\hline Lung AVM + upper GI angiodysplasia & 12 & 1.0 & 9 & 0.000 & 39,992 \\
\hline Lung AVM + angiodysplasia of the intestine & 4 & 0.3 & 7 & 0.000 & 15,426 \\
\hline
\end{tabular}

AVM, arteriovenous malformation; GI, gastrointestinal; HHT, hereditary hemorrhagic telangiectasia; ICD-9-CM, International Classification of Disease, 9th Revision, Clinical Modification.

Among people in the MarketScan 2010 databases who had one of these combinations of diagnoses, between 20 and $57 \%$ had HHT. These six combinations were reported for 111 (9.2\%) of the 1,203 people with HHT, and for $454(0.001 \%)$ persons without HHT diagnosis codes.

The four other combinations of signs shown in Table 4 were more than 2,000 times more frequently observed in people with HHT than in the general population without an HHT diagnosis code, with positive predictive values of $7-12 \%$.

\section{DISCUSSION}

The administrative prevalence of HHT, 0.3 per 10,000 US residents with employer-sponsored health insurance, is substantially lower than estimates of 1.5-2 per 10,000 based on active, population-based surveillance studies conducted in Europe and Asia. ${ }^{4,5}$ This low frequency is consistent with previously reported US findings of a low degree of clinical recognition and reporting of $\mathrm{HHT}^{18}$ and hence is not surprising. A diagnosis of HHT requires that multiple criteria be met, including a family history of symptoms. ${ }^{14}$

At older ages, the frequency of HHT using the study case definition approaches the expected population frequency. Assuming that the presence of an ICD-9 code for 448.0 reflects a medical diagnosis of HHT, the majority of older adults with HHT are clinically recognized. However, late diagnosis of HHT is insufficient. Two older studies reported that HHT was associated with significantly elevated mortality below the age of 60 years; ${ }^{4,13}$ more recent data are lacking. Timely diagnosis enables appropriate clinical management of HHT and prevention of life-threatening complications. For example, preventative embolization of lung AVMs has been projected in a decision analysis based on clinical evidence to improve both life expectancy and health-related quality of life. ${ }^{19}$ Therefore, reducing underdiagnosis of HHT is a matter of public health importance.

The major potential contribution of the current study is the development of an approach to identify patients with combinations of signs or characteristics of HHT in order to refer them for evaluation of potential HHT. The study has identified six combinations of signs or symptoms that appear to be highly specific to the presence of billing codes for HHT, each of which occurs more than 2,000 times as frequently in people with presumed HHT (Table 4). Further study is warranted.

The reliance on administrative data and ICD-9 codes poses multiple limitations, and further investigation is warranted based on record linkage of administrative claims data for individuals and clinical records to validate the use of the 448.0 ICD-9-CM code for HHT. First, it is not known to what extent individuals with a medical diagnosis of HHT or with clinical suspicion of HHT have the 448.0 code listed. Second, it is possible that individuals meeting the study case definition may not actually have HHT.

Another limitation is that the signs or complications of HHT may not be reported in claims records. Conditions such as epistaxis or telangiectases may not be considered major and hence are not likely to be recorded for billing purposes. Serious complications such as AVMs are likely to be recorded while being actively managed. If an AVM is successfully embolized, it may not be recorded in subsequent years. Consequently, it is not surprising that the frequency with which classic signs of HHT are recorded in claims for people with HHT coded is substantially less than expected based on clinical data. In particular, lung or pulmonary AVMs, many of which are asymptomatic, are reported to occur in up to $50 \%$ of people with HHT1 and $10-25 \%$ of people with HHT2 ${ }^{8}$ In the United States, HHT1 is slightly more common than HHT2 ${ }^{6,9}$ In the current study, 9\% of people with HHT (13\% among those aged $30-59$ years) had a diagnosis of lung AVM recorded. In addition, the current study is representative of the US health-care system, and patients not seen at HHT centers may be less likely to have AVMs diagnosed and treated.

Another potential concern is that the 448.0 code does not necessarily indicate the presence of a medical diagnosis of 
HHT. In particular, not everyone aged 70 years or older meeting the case definition for HHT may have had HHT medical diagnoses. Fewer older adults with coded HHT had signs specific to HHT, e.g., epistaxis, telangiectasia, or AVMs, recorded in claims, and the majority had GI bleeding, anemia, or both as the main clinical signs. According to Canadian clinical data, anemia is found in $25 \%$ of HHT patients and $15 \%$ of non-HHT patients, a modest excess..$^{20}$ By contrast, in the current study, anemia was recorded as a diagnosis among $49 \%$ of presumed HHT patients aged $30-59$ years (Table 3 ) as compared with $5 \%$ of non-HHT patients (data not shown). Further investigation is warranted to investigate whether the 448.0 code may be used in some cases as a billing code in patients with unexplained GI bleeding and anemia. To validate the use of the 448.0 ICD9-CM code to ascertain cases of HHT, it is necessary to make a record linkage of administrative data for individuals with validated clinical HHT diagnoses.

This study has a number of other limitations. Some of those limitations are specific to the proprietary claims databases used, which comprise convenience samples of individuals with employer-sponsored insurance and are not representative of the US population. The only demographic variables available were age and sex. Race and ethnicity are not routinely collected by health plans in the United States unless directed by public payers and hence that information is not contained in the MarketScan Commercial or Medicare Supplemental databases.

On the other hand, claims data are superior to hospital discharge data for HHT surveillance. Although national hospital discharge data are representative of the entire population and include information on race/ethnicity, HHT is managed on an outpatient basis. According to the US Agency for Healthcare Research and Quality's Health Care Utilization Project Nationwide Inpatient Sample of hospital inpatient discharges, during 1997 through 2010, there were an average of 3,5004,000 discharges per year with a code of 448.0 listed (source: http://www.hcupnet.ahrq.gov/). This represents an annual HHT-associated hospitalization rate of a little more than 0.01 per 10,000 population.

More generally, the reliance on ICD codes in administrative data for case ascertainment has implications for the potential utility of such data for health services research on rare disorders. ${ }^{1,3}$ Although administrative data are useful for research on disorders such as HHT or amyotrophic lateral sclerosis, for which there are highly specific ICD codes ${ }^{21}$ the majority of rare disorders share codes with other, similar disorders. For example, although most people with Duchenne-Becker muscular dystrophy typically have health records listing one of two ICD codes, the majority of people who have those codes may have a different form of muscular dystrophy with different etiology, epidemiology, and symptoms. ${ }^{22}$

A second limitation of administrative data is that medical information is not necessarily reported. For example, epistaxis is uncommonly recorded in billing records, probably because no medical treatment was prescribed. Of particular importance, it is not possible to reliably distinguish the presence of multiple
AVMs from single AVMs in administrative data. Although HHT is not a common cause of brain AVMs overall, it is a leading cause of multiple brain AVMs. In a study of patients with brain AVMs, multiple AVMs were detected in 39\% of those with HHT and only $1 \%$ of those without $\mathrm{HHT}^{23}$ Consequently, individuals with multiple brain AVMs should be regarded as candidates for a work-up for HHT, the same as patients with both brain and lung AVMs.

Prospective studies are needed to validate these algorithms for case finding. An integrated health-care system's database could be used to identify members with signs characteristic of HHT and to refer them for clinical evaluation. In addition, information contained in electronic medical records, such as the presence of multiple brain AVMs, could be used to improve positive predictive value. Although such an approach would not directly identify most individuals with undiagnosed HHT, it could identify index cases in many unrecognized HHT families. Once one member of a kindred is identified as having HHT and the causative genotype is found, it is straightforward and recommended to offer targeted molecular testing for the family-specific mutation to all first-degree relatives, ${ }^{14} 50 \%$ of whom on average will also be affected by HHT. Such cascade testing of relatives can allow diagnosis in an entire family, followed by recommended screening and management. ${ }^{24,25}$

\section{ACKNOWLEDGMENTS}

We acknowledge helpful comments received on earlier versions of this article from Barbara Bernhardt and Mike Soucie and comments from Jonathan Zaroff. The findings and conclusions in this report are those of the authors and do not necessarily represent the official position of the Centers for Disease Control and Prevention.

\section{DISCLOSURE}

The senior author, M.E.F., is medical director of the HHT Foundation International, which is a nonprofit patient support and advocacy organization. S.D.G. received travel support from the HHT Foundation International in connection with presentation of the findings of an earlier version of the analysis at the 9th HHT Scientific Conference, 20-24 May 2011, in Kemer, Turkey. The other authors declare no conflict of interest.

\section{REFERENCES}

1. Grosse SD, James AH, Lloyd-Puryear MA, Atrash HK. A public health framework for rare blood disorders. Am J Prev Med 2011;41(6 suppl 4):S319-S323.

2. Soucie JM, Nuss R, Evatt B, et al. Mortality among males with hemophilia: relations with source of medical care. The Hemophilia Surveillance System Project Investigators. Blood 2000;96:437-442.

3. Grosse SD, Boulet SL, Amendah DD, Oyeku SO. Administrative data sets and health services research on hemoglobinopathies: a review of the literature. Am J Prev Med 2010;38(suppl 4):S557-S567.

4. Kjeldsen AD, Vase P, Green A. Hereditary haemorrhagic telangiectasia: a population-based study of prevalence and mortality in Danish patients. J Intern Med 1999;245:31-39

5. Dakeishi M, Shioya T, Wada Y, et al. Genetic epidemiology of hereditary hemorrhagic telangiectasia in a local community in the northern part of Japan. Hum Mutat 2002;19:140-148.

6. McDonald J, Damjanovich K, Millson A, et al. Molecular diagnosis in hereditary hemorrhagic telangiectasia: findings in a series tested simultaneously by sequencing and deletion/duplication analysis. Clin Genet 2011;79:335-344. 
7. McDonald J, Bayrak-Toydemir P, Pyeritz RE. Hereditary hemorrhagic telangiectasia: an overview of diagnosis, management, and pathogenesis. Genet Med 2011;13:607-616.

8. Cottin V, Khouatraa C, Dupuis-Girod S, Cordiera JF. Pulmonary vascular disorders in hereditary hemorrhagic telangiectasia. In: Humbert M, Souza R, Simonneau G (eds). Pulmonary Vascular Disorders, vol. 41. Prog Respir Res. Karger: Basel, 2012:262-275.

9. Bayrak-Toydemir P, McDonald J, Markewitz B, et al. Genotype-phenotype correlation in hereditary hemorrhagic telangiectasia: mutations and manifestations. Am J Med Genet A 2006;140:463-470.

10. Letteboer TG, Mager JJ, Snijder RJ, et al. Genotype-phenotype relationship in hereditary haemorrhagic telangiectasia. J Med Genet 2006;43:371-377.

11. Shovlin CL. Hereditary haemorrhagic telangiectasia: pathophysiology, diagnosis and treatment. Blood Rev 2010;24:203-219.

12. Pierucci $P$, Lenato $G M$, Suppressa $P$, et al. A long diagnostic delay in patients with hereditary haemorrhagic telangiectasia: a questionnaire-based retrospective study. Orphanet J Rare Dis 2012;7:33.

13. Sabbà C, Pasculli $G$, Suppressa P, et al. Life expectancy in patients with hereditary haemorrhagic telangiectasia. QJM 2006;99:327-334.

14. Faughnan ME, Palda VA, Garcia-Tsao G, et al.; HHT Foundation International - Guidelines Working Group. International guidelines for the diagnosis and management of hereditary haemorrhagic telangiectasia. J Med Genet 2011:48:73-87.

15. Shovlin CL, Guttmacher AE, Buscarini E, et al. Diagnostic criteria for hereditary hemorrhagic telangiectasia (Rendu-Osler-Weber syndrome). Am J Med Genet 2000;91:66-67.

16. Li T, Carls GS, Panopalis P, Wang S, Gibson TB, Goetzel RZ. Long-term medical costs and resource utilization in systemic lupus erythematosus and lupus nephritis: a five-year analysis of a large medicaid population. Arthritis Rheum 2009;61:755-763.

17. Amendah DD, Mvundura M, Kavanagh PL, Sprinz PG, Grosse SD. Sickle cell disease-related pediatric medical expenditures in the U.S. Am J Prev Med 2010;38(suppl 4):S550-S556.

18. Guttmacher AE, Marchuk DA, White RI Jr. Hereditary hemorrhagic telangiectasia. N Engl J Med 1995;333:918-924.

19. Gupta S, Faughnan ME, Bayoumi AM. Embolization for pulmonary arteriovenous malformation in hereditary hemorrhagic telangiectasia: a decision analysis. Chest 2009;136:849-858.

20. Rozenberg D, Faughnan ME. Prevalence and nature of dyspnea in patients with hemorrhagic hereditary telangiectasia (HHT). Chest 2010; 138:841A.

21. Pisa FE, Verriello L, Deroma L, et al. The accuracy of discharge diagnosis coding for amyotrophic lateral sclerosis in a large teaching hospital. Eur J Epidemiol 2009;24:635-640

22. Ouyang L, Grosse SD, Kenneson A. Health care utilization and expenditures for children and young adults with muscular dystrophy in a privately insured population. J Child Neurol 2008:23:883-888

23. Bharatha A, Faughnan ME, Kim H, et al. Brain arteriovenous malformation multiplicity predicts the diagnosis of hereditary hemorrhagic telangiectasia: quantitative assessment. Stroke 2012;43:72-78.

24. Bernhardt BA, Zayac C, Trerotola SO, Asch DA, Pyeritz RE. Cost savings through molecular diagnosis for hereditary hemorrhagic telangiectasia. Genet Med 2012:14:604-610.

25. Bernhardt BA, Zayac C, Pyeritz RE. Why is genetic screening for autosomal dominant disorders underused in families? The case of hereditary hemorrhagic telangiectasia. Genet Med 2011;13:812-820. 